\title{
Wideband Metamaterial Absorber Based in Combination of Unit Cells
}

\author{
Puji Handayani \\ Department of Electrical Engineering \\ Institut Teknologi Sepuluh Nopember \\ Surabaya, Indonesia \\ puji@ee.its.ac.id
}

Gamantyo Hendrantoro

Department of Electrical Engineering

Institut Teknologi Sepuluh Nopember

Surabaya, Indonesia

gamantyo@ee.its.ac.id

\author{
Eko Setijadi \\ Department of Electrical Engineering \\ Institut Teknologi Sepuluh Nopember \\ Surabaya, Indonesia \\ ekoset@ee.its.ac.id
}

\author{
M. Rendy Anggara \\ Department of Electrical Engineering \\ Institut Teknologi Sepuluh Nopember \\ Surabaya, Indonesia \\ rendyanggara17@gmail.com \\ Ahmad Maulidiyanto \\ Department of Electrical Engineering \\ Institut Teknologi Sepuluh Nopember \\ Surabaya, Indonesia \\ maulud@ee.its.ac.id
}

\begin{abstract}
Metamaterial absorber is an electromagnetic wave absorber made from metamaterial. Especially, it works in narrow-band frequency as it is designed in a particular shape that related to its resonance frequency. However, some applications, e.g., anechoic chamber, requires metamaterial absorber that can work in a wide frequency band. This paper discussed the design of a wideband metamaterial absorber using the combination of multiple unit cells. The unit cell type was split-ring resonator (SRR). SRR had advantages in terms of its simple shape, it could have more than one resonant frequencies depending on the number of its ring, and its shape could be modified easily to obtain the desired resonant frequencies. We designed metamaterial absorber having a good absorption rate in $2-10 \mathrm{GHz}$ frequency band. To cover this wide frequency band, we used five unit cells which were arranged on a flat plane. Each unit cell had several resonant frequencies. The design was carried out using the simulation software of CST (Computer Simulation System). The fabricated design was measured and the results have shown that it had an absorption rate of $99 \%$ in the measured frequency band.
\end{abstract} rate

Keywords-metamaterial absorber, unit cell, an absorption

\section{INTRODUCTION}

Electromagnetic waves absorber is a component needed to suppress interference between electronic components or microwave components. In measuring an antenna in a room or building, an absorber is needed to prevent the reflection of electromagnetic waves by the walls of the measurement room and also to prevent interference from outside the room, so that the condition of the measurement room qualifies as free space. The material of absorber has evolved to the use of metamaterials. The metamaterial is a structure of artificial material. It can be represented by the complex values of electric permittivity and magnetic permeability. These parameters can be independently controlled by varying the shape and dimensions of the structure. By tuning the electric and magnetic resonances, a metamaterial can be impedancematched to $\mathrm{fr} f f$ ee space [1].

Metamaterial absorber has several advantages compared to the conventional one. The metamaterial absorber is much thinner than the traditional absorber which has a quarter wavelength thickness. Moreover, metamaterial manufacturing techniques are low costs. However, most of metamaterial absorber structures work based on a strong electromagnetic resonance that absorbs the coming electromagnetic wave. Therefore, its performance in terms of absorbance bandwidth is quite narrow [2].

Meanwhile, for an anechoic chamber application, an absorber that has a wide enough bandwidth is needed. One technique to widen the bandwidth of metamaterial absorbers is to create a structure consisting of a combination of several unit cells, where each of its works at a certain resonant frequency. This structure will have an absorption rate as a superposition of the absorption rates of all unit cells [2]. Besides that, in [3], a unit cell was composed of two SRRs. This structure produces two resonant frequencies. This paper propose the design of metamaterial absorber that can be used as an absorber for the anechoic chamber for the $2.4-9.6 \mathrm{GHz}$ band or occupies a portion of the S, C, and part of the $\mathrm{X}$ band. The desired bandwidth is far wider than the bandwidth in [2] and [3]. Therefore, even though the basic technique used is the same, we have designed some unit cells that have several adjacent resonant frequencies and construct a single structure made from combination of these unit cells to cover the overall desired bandwidth. The design of the metamaterial structure was carried out using MWS-CST (Micro Wave Studio-Computer Simulation System), a commercial simulation software.

The following Section II described all unit cells we have designed and their performances, whereas in Section III we confirm these with the measurement.

\section{SRR UNIT CELl SIMULATION}

First, The unit cell of the metamaterial absorber that has been designed as a ring base shape, called a split-ring resonator (SRR). SRR was introduced by Pendry [4]. SRR has a pair of rings of a certain thickness made of metal etched on a dielectric substrate. The two rings have opposite gaps. The resonant frequency of SRR is determined by the SRR dimensions and relative permittivity of the substrate used. The SRR dimension for a particular resonant frequency $f_{0}$ is calculated using the following equation [5],

$$
f_{0} \approx \frac{c}{\pi\left(R_{1}+R_{2}\right)}\left(\frac{1+\varepsilon_{r}}{2 \varepsilon_{r}}\right)^{\frac{1}{2}}
$$


where $c$ is the speed of light in free space, $R_{1}$ and $R_{2}$ denote the radius of the outer and inner ring as depicted in Figure 1, respectively, and $\varepsilon_{\mathrm{r}}$ is the relative permittivity of the substrate. In Figure 1, $g$ is the gap of the ring.

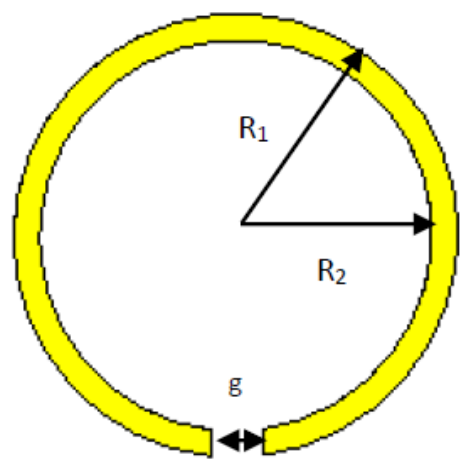

Fig. 1. SRR basic geometry

The initial design of the ring size was determined using (1). Referring the work on [6], to get resonances at the desired frequencies, ring size optimization was carried out by performing sweeping parameters. And as can be seen in (1), gap-width $g$ in Fig. 1 was not written explicitly. Generally, gap-width produces capacitance that affect resonant frequency. To determine the gap-width in accordance with the desired resonant frequency, we also apply 'parameter sweep' of gap-width in simulation as well as we run the 'parameter sweep' of other parameters.

We have designed a wideband absorber by combining 5 types of unit cells, where each of these unit cells works on a specific bandwidth. The unit cells are Ring4\#1, Ring4\#2, Ring3\#1, Ring3\#2, and Small Ring. The geometry of Ring4, Ring3, and Small Ring unit cells can be seen in Fig. 2. Small rings have the same structure as Ring3, consisting of three circles smaller than the Ring 3 circle. The parameters of each unit cell was obtained using (1) and by applying sweeping parameters for each SRR parameters to optimize the desired resonant frequencies. The size of each ring of the five types of a unit cell after optimization was shown in Table I, Table II, and Table III.

TABLE I. THE SIZE OF RING4

\begin{tabular}{|c|c|c|c|}
\hline \multicolumn{2}{|c|}{ Ring4\#1 } & \multicolumn{2}{c|}{ Ring4\#2 } \\
\hline Parameters & Radius $(\boldsymbol{m m})$ & Parameters & Radius $(\boldsymbol{m m})$ \\
\hline $\mathrm{R} 1 \mathrm{i}$ & 13.6 & $\mathrm{R} 1 \mathrm{i}$ & 13.1 \\
\hline $\mathrm{R} 1 \mathrm{o}$ & 15.6 & $\mathrm{R} 1 \mathrm{o}$ & 15.6 \\
\hline $\mathrm{g} 1$ & 2.8 & $\mathrm{~g} 1$ & 5 \\
\hline $\mathrm{R} 2 \mathrm{i}$ & 9 & $\mathrm{R} 2 \mathrm{i}$ & 9.2 \\
\hline $\mathrm{R} 2 \mathrm{O}$ & 12 & $\mathrm{R} 2 \mathrm{O}$ & 11.8 \\
\hline $\mathrm{g} 2$ & 1.2 & $\mathrm{~g} 2$ & 1 \\
\hline $\mathrm{R} 3 \mathrm{i}$ & 6.5 & $\mathrm{R} 3 \mathrm{i}$ & 6.5 \\
\hline $\mathrm{R} 30$ & 8 & $\mathrm{R} 3 \mathrm{o}$ & 8 \\
\hline $\mathrm{g} 3$ & 0.6 & $\mathrm{~g} 3$ & 1 \\
\hline $\mathrm{R} 4 \mathrm{i}$ & 4.3 & $\mathrm{R} 4 \mathrm{i}$ & 4.2 \\
\hline $\mathrm{R} 4 \mathrm{o}$ & 5.6 & $\mathrm{R} 4 \mathrm{o}$ & 5.6 \\
\hline $\mathrm{g} 4$ & 0.6 & $\mathrm{~g} 4$ & 0.6 \\
\hline
\end{tabular}

TABLE II. THE SIZE OF RING3

\begin{tabular}{|c|c|c|c|}
\hline \multicolumn{2}{|c|}{ Ring311 } & \multicolumn{2}{c|}{ Ring3\#2 } \\
\hline Parameter & Radius $(\mathbf{m m})$ & Parameter & Radius $(\mathbf{m m})$ \\
\hline $\mathrm{R} 1 \mathrm{i}$ & 14.6 & $\mathrm{R} 1 \mathrm{i}$ & 14.6 \\
\hline $\mathrm{R} 1 \mathrm{o}$ & 15.6 & $\mathrm{R} 1 \mathrm{o}$ & 15.6 \\
\hline $\mathrm{g} 1$ & 1 & $\mathrm{~g} 1$ & 1 \\
\hline $\mathrm{R} 2 \mathrm{i}$ & 11.2 & $\mathrm{R} 2 \mathrm{i}$ & 11.2 \\
\hline $\mathrm{R} 2 \mathrm{o}$ & 13.2 & $\mathrm{R} 2 \mathrm{o}$ & 13.2 \\
\hline $\mathrm{g} 2$ & 0.8 & $\mathrm{~g} 2$ & 0.8 \\
\hline $\mathrm{R} 3 \mathrm{i}$ & 8.3 & $\mathrm{R} 3 \mathrm{i}$ & 8.3 \\
\hline $\mathrm{R} 30$ & 10 & $\mathrm{R} 3 \mathrm{o}$ & 10 \\
\hline $\mathrm{g} 3$ & 0.7 & $\mathrm{~g} 3$ & 0.7 \\
\hline
\end{tabular}

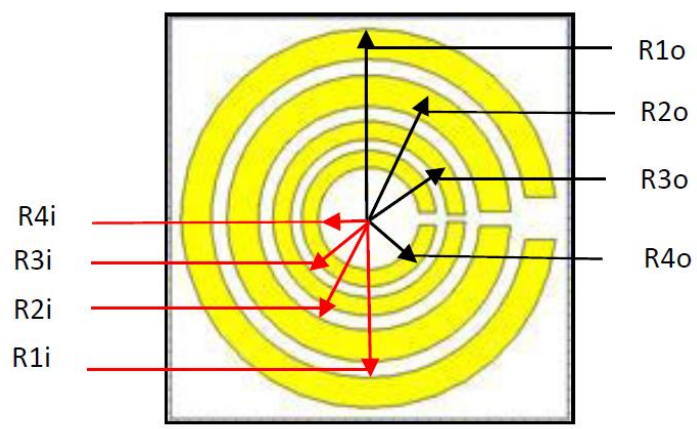

Fig. 2. SRR basic geometry

TABLE III. THE SIZE OF SMALL RING

\begin{tabular}{|c|c|}
\hline Parameter & Radius $(\mathbf{m m})$ \\
\hline R1i & 5.8 \\
\hline R1o & 6.2 \\
\hline g1 & 0.7 \\
\hline R2i & 5 \\
\hline R2o & 5.4 \\
\hline g2 & 1.2 \\
\hline R3i & 4.1 \\
\hline R3o & 4.5 \\
\hline g3 & 1.2 \\
\hline
\end{tabular}

Ring4 was originally designed to absorb waves at the frequency of $2.6 \mathrm{GHz}, 3.5 \mathrm{GHz}, 4.2 \mathrm{GHz}$, and 7.2 GHz. The simulation result of S11 parameter of this unit cell which was called Ring4\#1 was depicted in Figure 3. Ring4\#2 is a modification of Ring4\#1 to shift the resonant frequencies, so that new resonant frequencies will be obtained near the resonant frequencies of Ring4\#1, and the simulation result could be seen in Figure 4. Thus if Ring4\#1 and Ring4\#2 were combined the new structure will expand the bandwidth of Ring4.

From the simulation results of Ring 3 and Ring 4, it was found that absorption in the frequency band above $5 \mathrm{GHz}$ was still low, as indicated by the value of S11 which was still quite high. To overcome this problem, a Small Ring unit cell was created. The Small Ring was designed to produce resonances at $6.2 \mathrm{GHz}, 7.8 \mathrm{GHz}$, and $9.4 \mathrm{GHz}$. The simulation result of $\mathrm{S} 11$ parameter of the Small Ring appeared in Figure 7. 


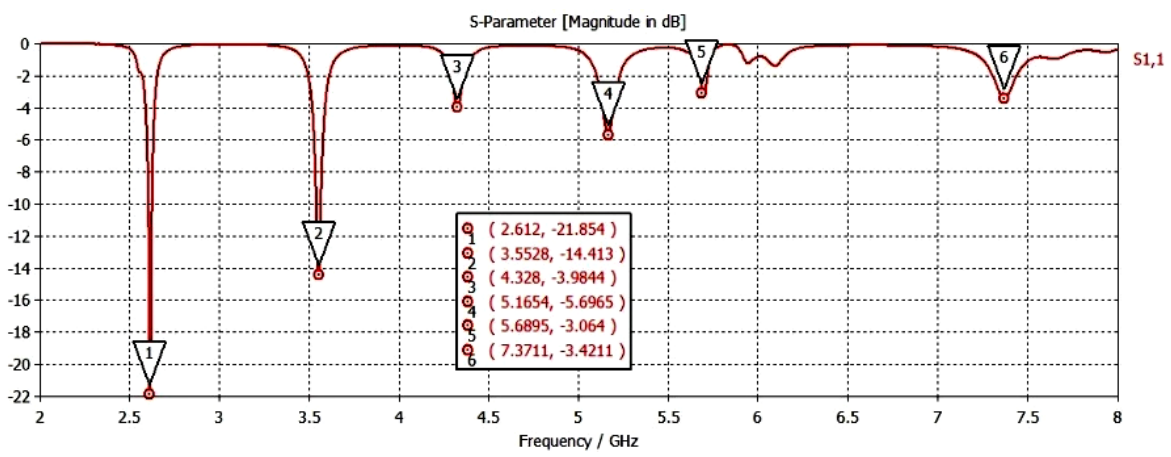

Fig. 3. The simulation result of S11 parameter of Ring4\#1

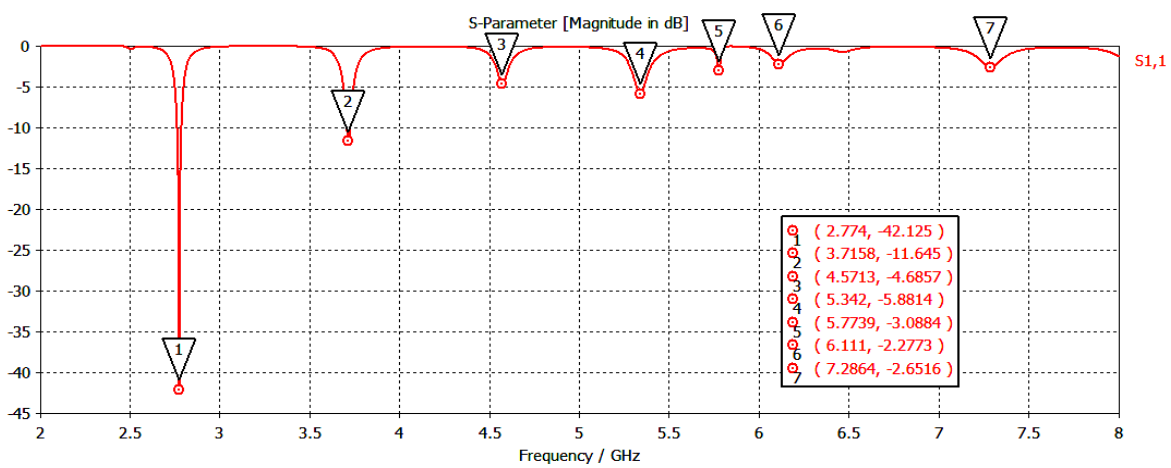

Fig. 4. The simulation result of S1 parameter of Ring4\#2

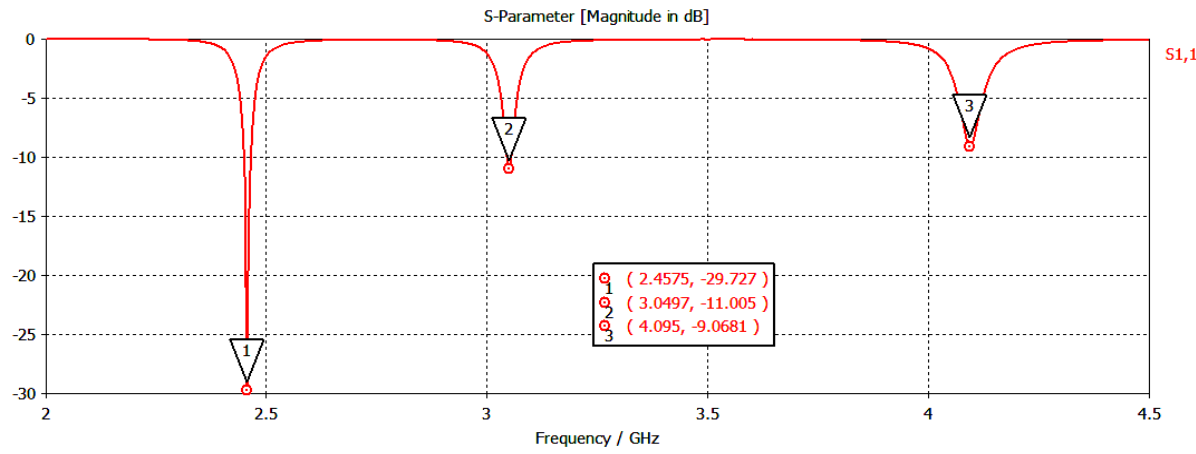

Fig. 5. The simulation result of S11 parameter of Ring3\#1

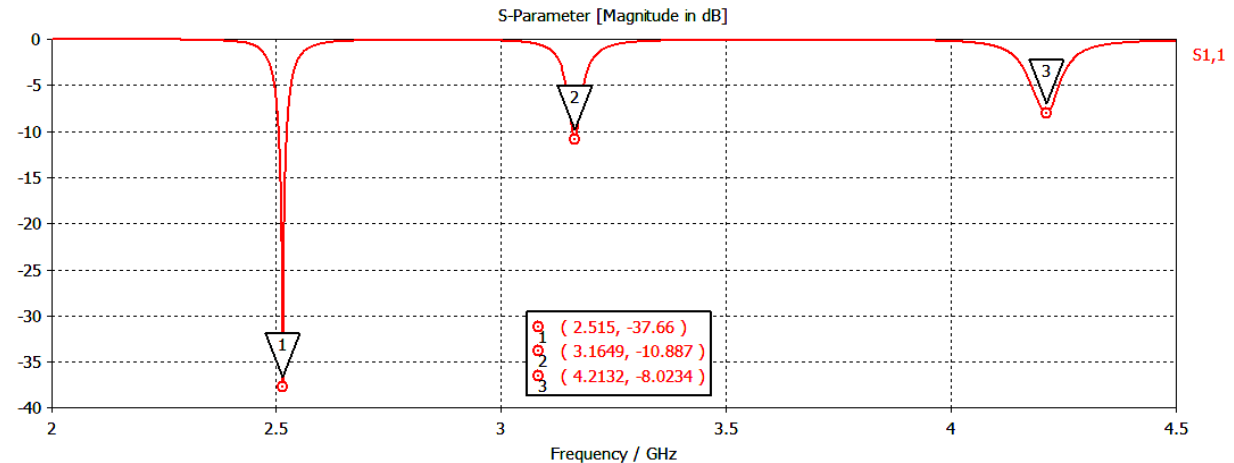

Fig. 6. The simulation result of S11 parameter of Ring3\#2 


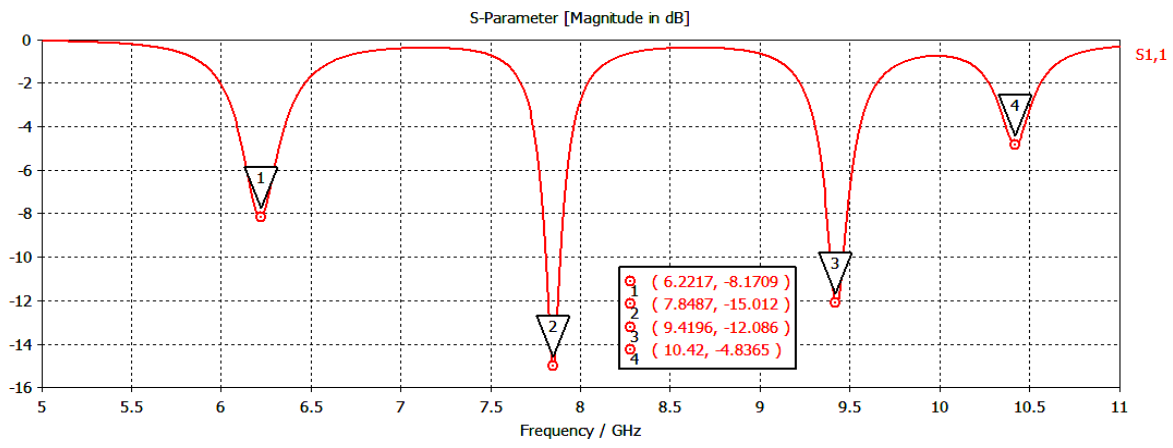

Fig. 7. The simulation result of S11 parameter of Small Ring

The five units cells that have been designed were then fabricated by the arrangement as shown in Figure 8 . The arrangement in Fig. 8 was based on the consideration that the absorber area can be occupied efficiently by the unit cells. This combined structure was expected to produce an absorber that works well at the desired resonant frequencies. The substrate used was FR4 with dimensions of $30 \times 30 \times 1.6 \mathrm{~mm}$. Thus there were 9 large unit cells and 8 small unit cells both in the vertical and horizontal direction. The backside of this structure was a copper surface which functions as a ground plane. The fabricated structure was shown in Figure 9.

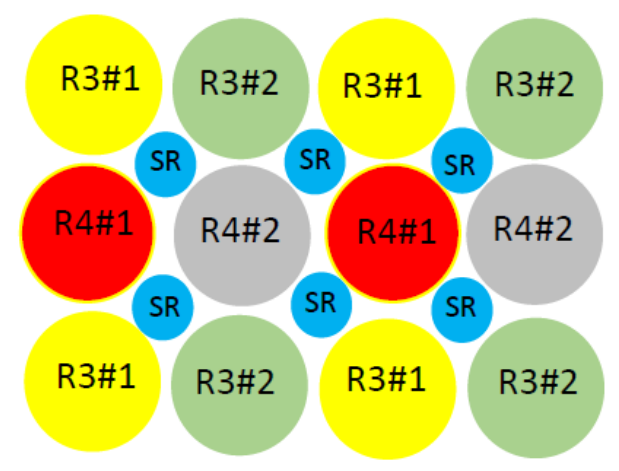

Fig. 8. Unit cell merges layouts. R3\#1, R3\#2, R4\#1, R4\#2, and SR stand for Ring3\#1, Ring3\#2, Ring4\#1, Ring4\#2, and Small Ring, respectively

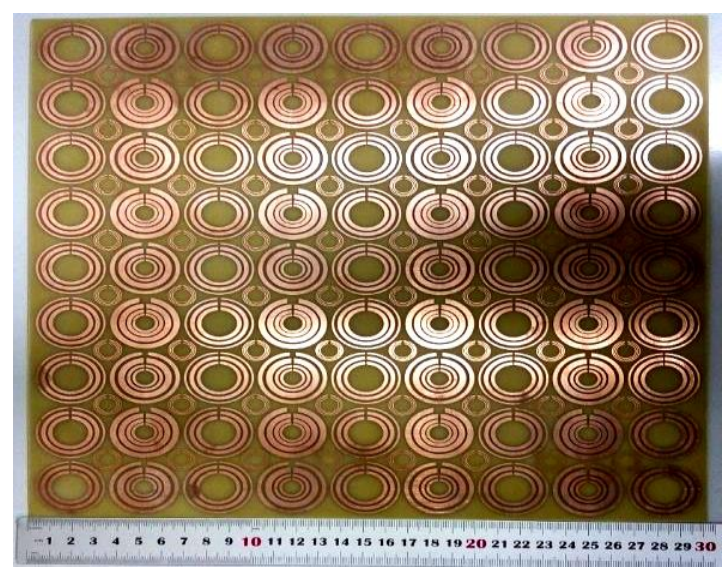

Fig. 9. The fabricated structure of wideband metamaterial absorber that has been designed

\section{Measurement Results}

Absorber performance was expressed by the absorption rate parameter. An absorption rate can be calculated based on S11 parameter as follows,

$$
A=1-\left|S_{11}\right|^{2}-\left|S_{21}\right|^{2}
$$

Because the back of the absorber was a conductor surface, S21 was zero. Then,

$$
A=1-\left|S_{11}\right|^{2}
$$

To get an absorption rate of at least $80 \%$, S11 should have the highest value of $-7 \mathrm{~dB}$.

We measured S11 parameter of the fabricated absorber using VNA. The measurement setup was shown in Figure 10. However, due to the limitations of the VNA frequency range, measurements are only made at 2.2-3.6 GHz. The measurement setup was shown in Figure 9. The distance between the horn antenna and absorber met the conditions of the antenna far-field region. We kept the position of the absorber was in the $3 \mathrm{~dB}$ beamwidth of the horn. The result was depicted in Figure 11. The figure shows that the absorber had an absorption rate of more than $80 \%$ in the measured frequency band. So, the measurement result met the simulation one in the measured frequency band.

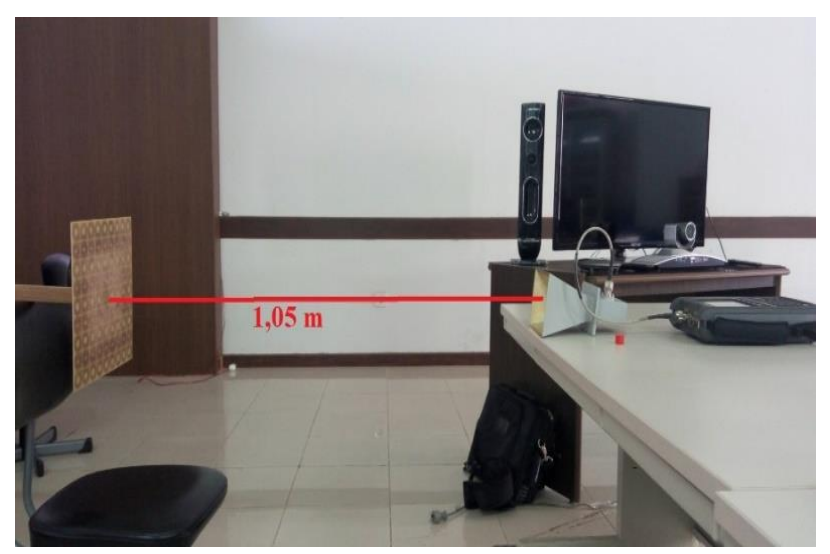

Fig. 10. The measurement setup 


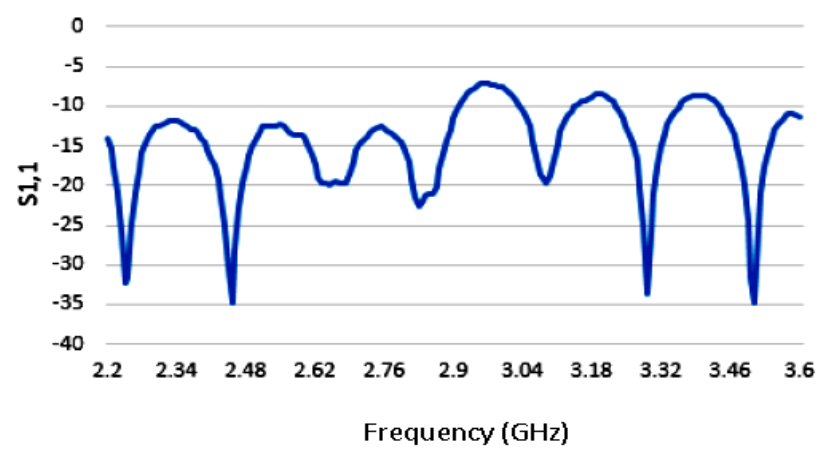

Fig. 11. The measurement setup

\section{CONCLUSION}

A broadband metamaterial absorber could be built by combining several unit cells. Each unit cell could be designed to have several resonant frequencies together by arranging several basic structures. In this study, the basic structure of SRR was used. To get bandwidth characteristics that are quite wide at each resonant frequency of the cell unit, the size of this cell unit could be modified to simply shift the resonant frequency that has been obtained. Thus the combination of the two cell units will produce a bandwidth that is wide enough at the desired resonant frequency. We have designed metamaterial absorber having good absorption rate in 2$10 \mathrm{GHz}$ frequency band by using combination of unit cells. To cover this wide frequency band, we used five unit cells which were arranged on a flat plane. The measurements results show that the absorber works well in the measured frequency band.

Given that there are many basic types of cell unit structures with various geometric shapes, the wideband metamaterial absorber design is very flexible to develop.

\section{REFERENCES}

[1] N. I. Landy, et al., "Perfect Metamaterial Absorber," Physical Review Letters, vol. 100, May 2008.

[2] Hong-Min Lee and Hyung-Sup Lee, "A Method for Extending the Bandwidth of Metamaterial Absorber," International Journal of Antennas and Propagation, vol. 2012, pp. 1-7.

[3] Chaurasiya, et al, "Dual-Band Polarization-Incentive Metamaterial Absorber With Bandwidth-Enhancement at $\mathrm{Ku}-$ band for EMI/EMC Application”, IEEE International Microwave and RF Conference, 2014.

[4] J. B. Pendry, et al., "Magnetism from Conductors and Enhanced Nonlinear Phenomena," IEEE Transactions on Microwave Theory and Techniques, vol. 47, pp. 2075-2084, Nov. 1999.

[5] Kin-Lu Wong, et al., "Printed Ring Slot Antenna for Circular Polarization," IEEE Transactions on Antennas and Propagation, vol. 50, pp. 75-77, Jan 2002.

[6] Somak Bhattacharyya, et al, "A Broadband Wide Angle Metamaterial Absorber for Defense Applications," IEEE International Microwave and RF Conference, 2014. 